\title{
Performance and Improvements of the ATLAS Jet Trigger System
}

Valerie Lang on Behalf of the ATLAS Collaboration

\section{Motivation for Triggering on Jets}

Jets are the most common physics objects which are produced at a hadron collider such as the LHC. With luminosities up to $3.65 \cdot 10^{33} \mathrm{~cm}^{-2} \mathrm{~s}^{-1}$ in 2011 and 7.73. $10^{33} \mathrm{~cm}^{-2} \mathrm{~s}^{-1}$ in 2012 as well as a bunch crossing rate of $20 \mathrm{MHz}$, the excellent performance of the jet trigger is fundamental to achieving the physics goals of the ATLAS experiment.

\section{Jet Signatures} Jets, consisting of varying numbers of hadrons from the fragmentation of highenergy quarks or gluons, leave both tracks in the inner detector and energy deposits in the calorimeter. The display shows an event with 9 jets above $55 \mathrm{GeV}$ and 7 jets even above $80 \mathrm{GeV}$.

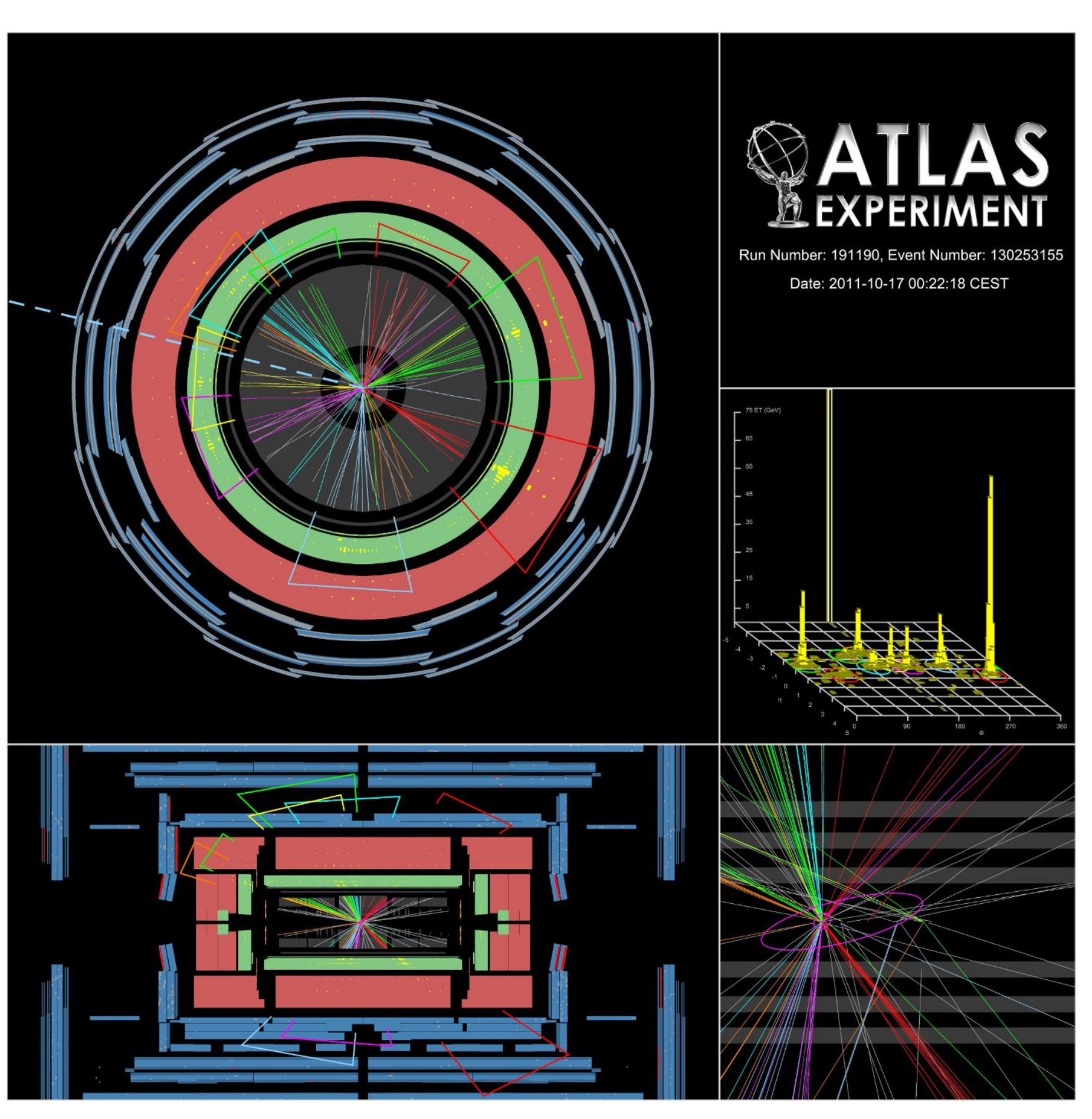

\section{ATLAS Jet Trigger System}

The ATLAS trigger system is designed in 3 levels: Level 1 (L1), Level 2 (L2) and Event Filter (EF). The jet trigger is an important part of the trigger and data acquisition system and is allocated $\sim 25-30 \%$ of the total bandwidth. Jet Trigger System

Improvements of the Jet Trigger System $20 \mathrm{MHz}$

$\downarrow \begin{array}{ll}\mathbf{L} 1 \begin{array}{l}\text { Sliding window algorithm } \\ \text { on calorimeter towers of } \\ 0.2 \times 0.2 \text { in } \eta \times \varphi\end{array} & \begin{array}{l}\text { Regions of } \\ \text { Interest } \\ \text { (Rol) }\end{array}\end{array}$

Simplified cone algorithm around L1

L2 Rol as seed using full calorimeter granularity 1 Offline anti- $k_{t}$ algorithm on nt building

EF topological clusters using entire event data and full calibration

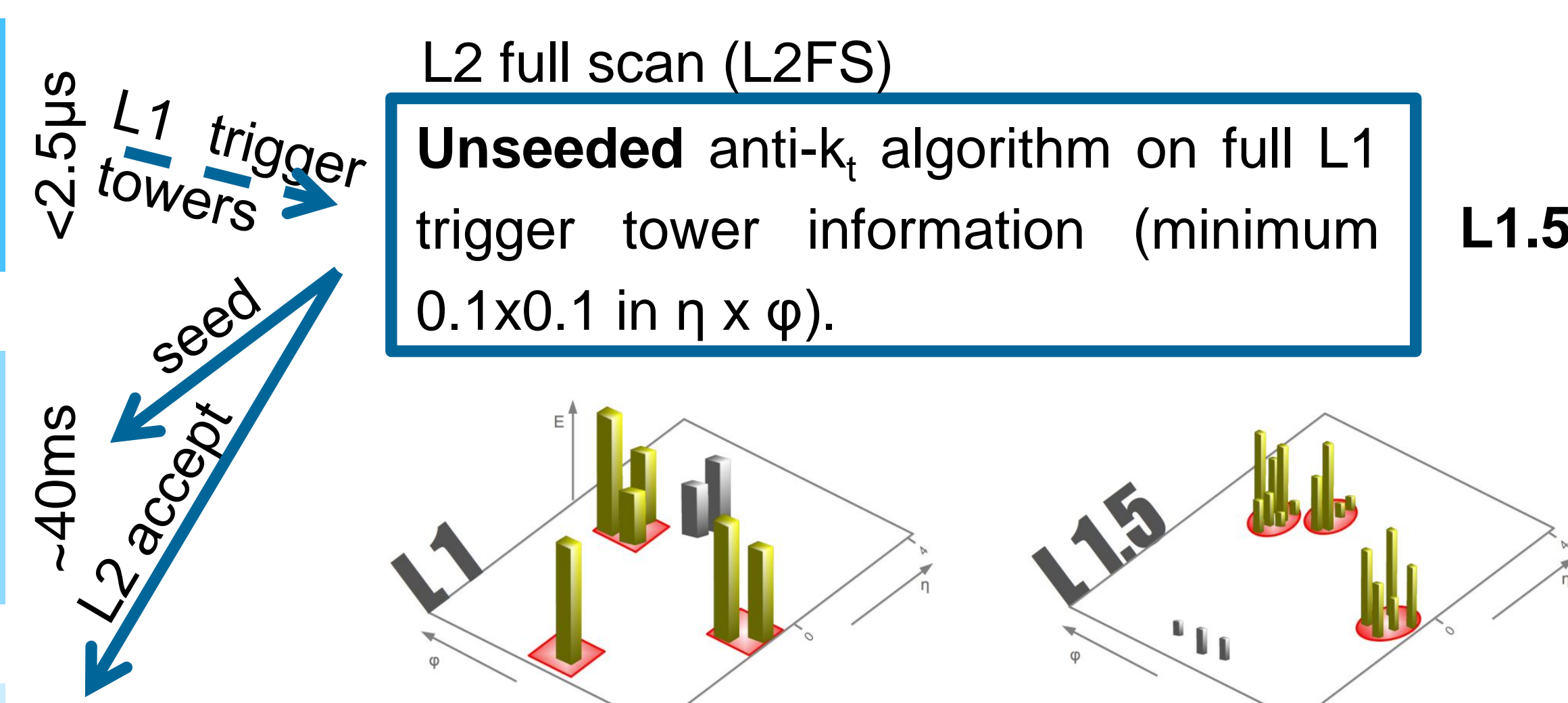

Full calorimeter unpacking at EF

Unseeded jet finding with the offline EF $^{\star}$ anti- $\mathrm{k}_{\mathrm{t}}$ algorithm on topological clusters

Inefficiencies of the Rol-based jet trigger approach in multi-jet topologies, especially for close-by jets, can be recovered with the L1.5 trigger and the unseeded jet finding at EF.

\section{Trigger Efficiency, Resolution and Performance of L2FS}

Rerunning L2 and EF (HLT) algorithms offline on data from proton-proton collisions with $\sqrt{ } \mathbf{s}=7 \mathrm{TeV}$ in 2011, allowed to study the performance of L2FS with respect to the usual L1 Rol-based L2 triggers.

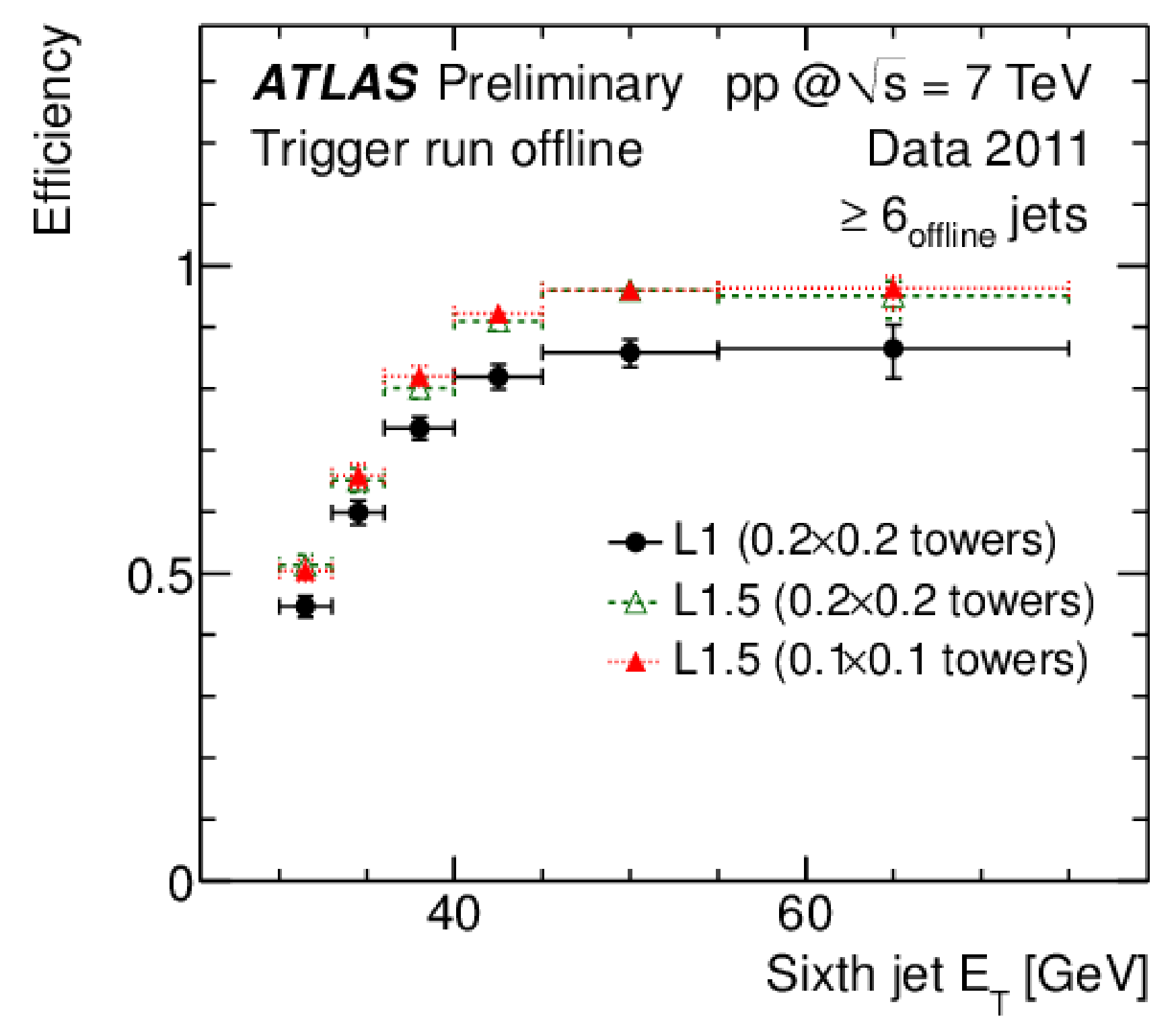

Efficiency for $L 1$ and L1.5 jets to satisfy a 6 -jet trigger in events with at least 6 offline jets with $|\eta|<2.8$ and $E_{T}>30 G e V$. At $L 1$, a $0.8 \times 0.8$ sliding window algorithm was used for jet finding. At $L 1.5$ as well as offline, the anti- $\mathrm{k}_{\mathrm{t}}$ algorithm with $\mathrm{R}=0.4$ was applied to either $0.2 \times 0.2$ or $0.1 \times 0.1 \mathrm{~L} 1$ trigger towers (L1.5) or using the full calorimeter granularity (offline).

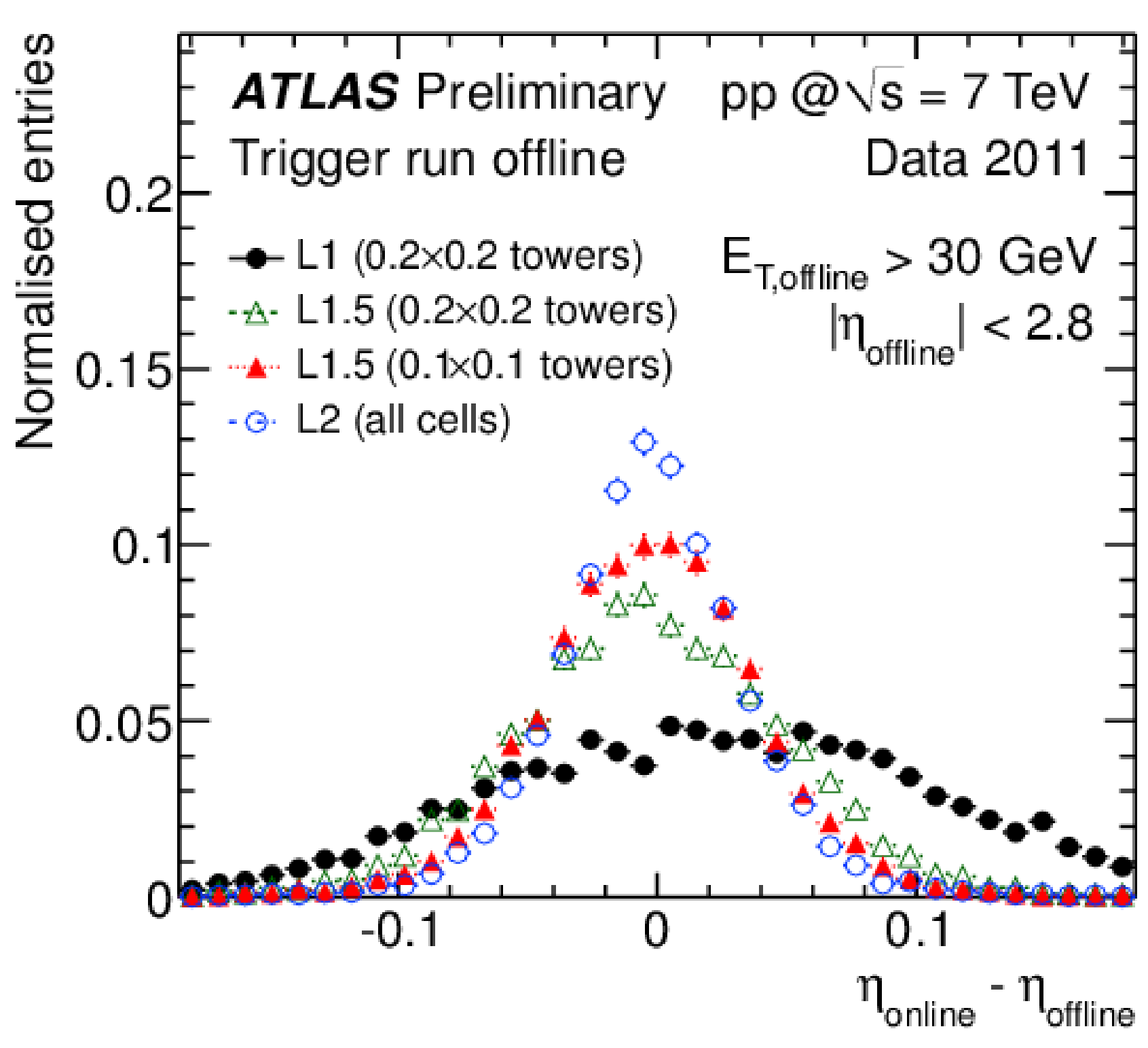

Jet position resolution in $\eta$ of the $L 1, L 1.5$ and $L 2$ jet algorithms. The jet finding algorithms are as before, $\mathrm{L} 2$ uses a 3-iteration cone with $R=0.4$ seeded by a $L 1$ jet. The offset towards positive $\Delta \eta$ at $L 1$ is an artefact of the position recording at $\mathrm{L} 1$.

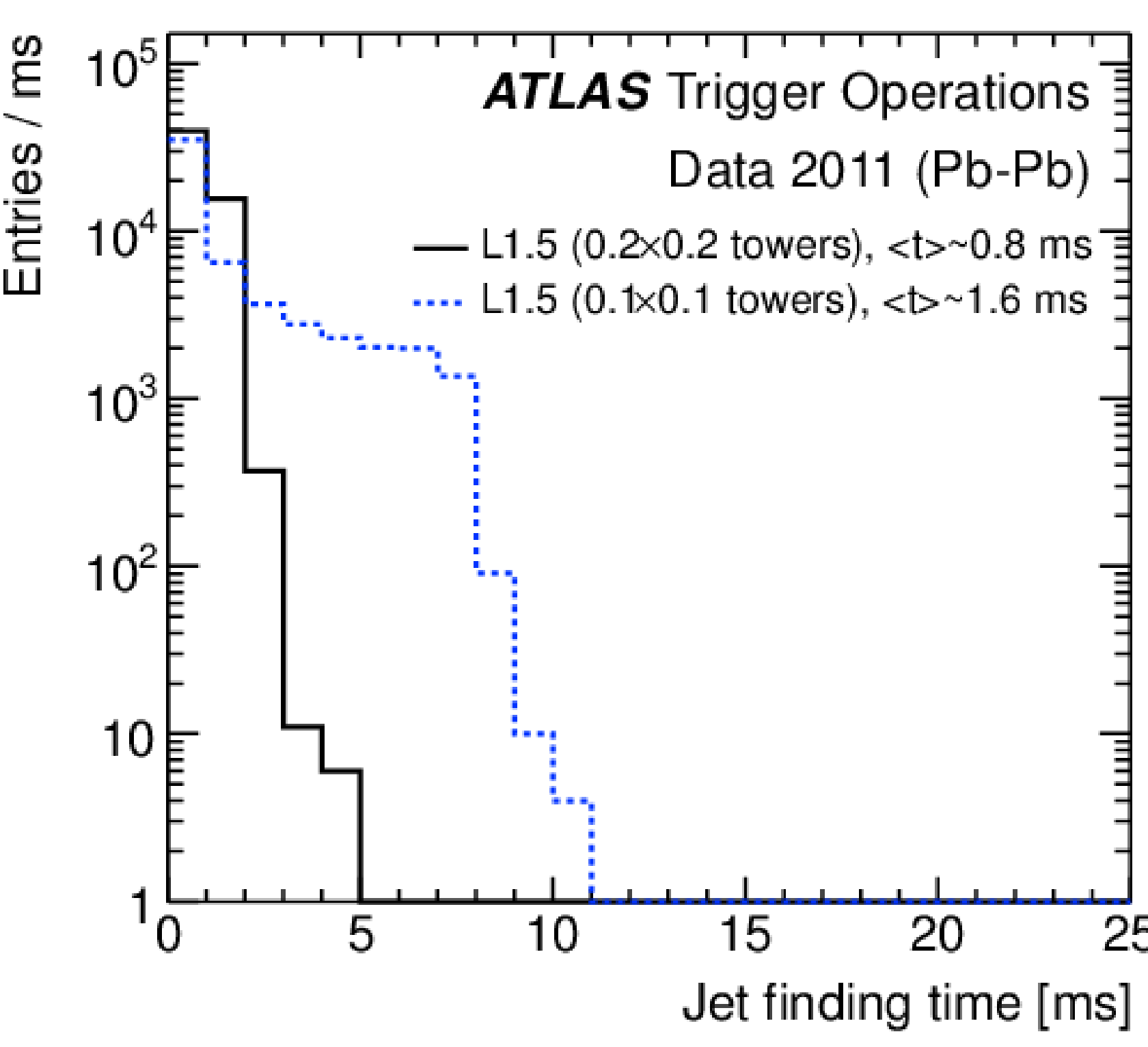

Time needed for jet finding at L1.5 when applying the anti- $\mathrm{k}_{\mathrm{t}}$ algorithm with $\mathrm{R}=0.4$ to $0.2 \times 0.2$ or $0.1 \times 0.1 \mathrm{~L} 1$ trigger towers. The nominal time limit for the $\mathrm{L} 2$ trigger is $40 \mathrm{~ms}$.

This timing was measured with the $2011 \mathrm{~Pb}-\mathrm{Pb}$ collisions at $\sqrt{\mathrm{s}_{\mathrm{NN}}}=2.76 \mathrm{TeV}$.

\section{Pile-up and Noise Suppression}

Multiple interactions, around 35 in 2012, can occur at the same crossing of proton bunches as the interesting event which triggers the readout. The recorded event consists of a superposition of the interesting collision with several additional pile-up interactions (in-time pile-up) and even signals from particles generated a few bunch crossings before or after (out-of-time pile-up). Since May 2011, a noise suppression tool implemented at L2 and EF considers electronics and pile-up noise. The combined noise level is used to determine the threshold energies for the calorimeter cells considered in the jet reconstruction, resulting in a more precise measurement of the jet energy, and thus improving the resolution and the efficiency turn-on of the jet triggers.

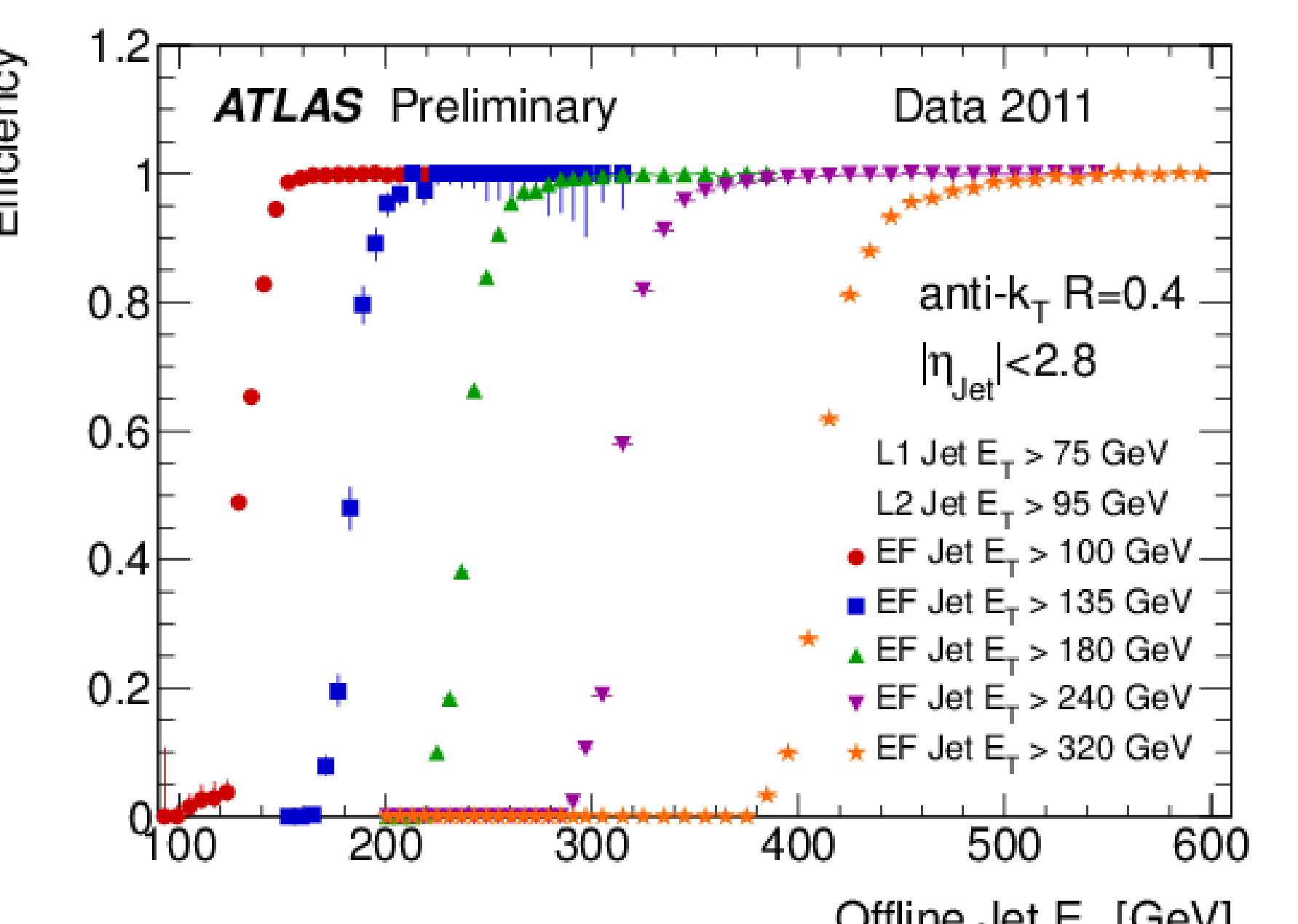

Trigger efficiencies of the EF inclusive jet trigger for 5 different choices of thresholds, using the full calorimeter scan at EF and the noise suppression at L2 and EF to improve jet finding and jet energy measurement. Jets at $\mathrm{EF}$ were reconstructed using the anti-k algorithm with $\mathrm{R}=0.4$.

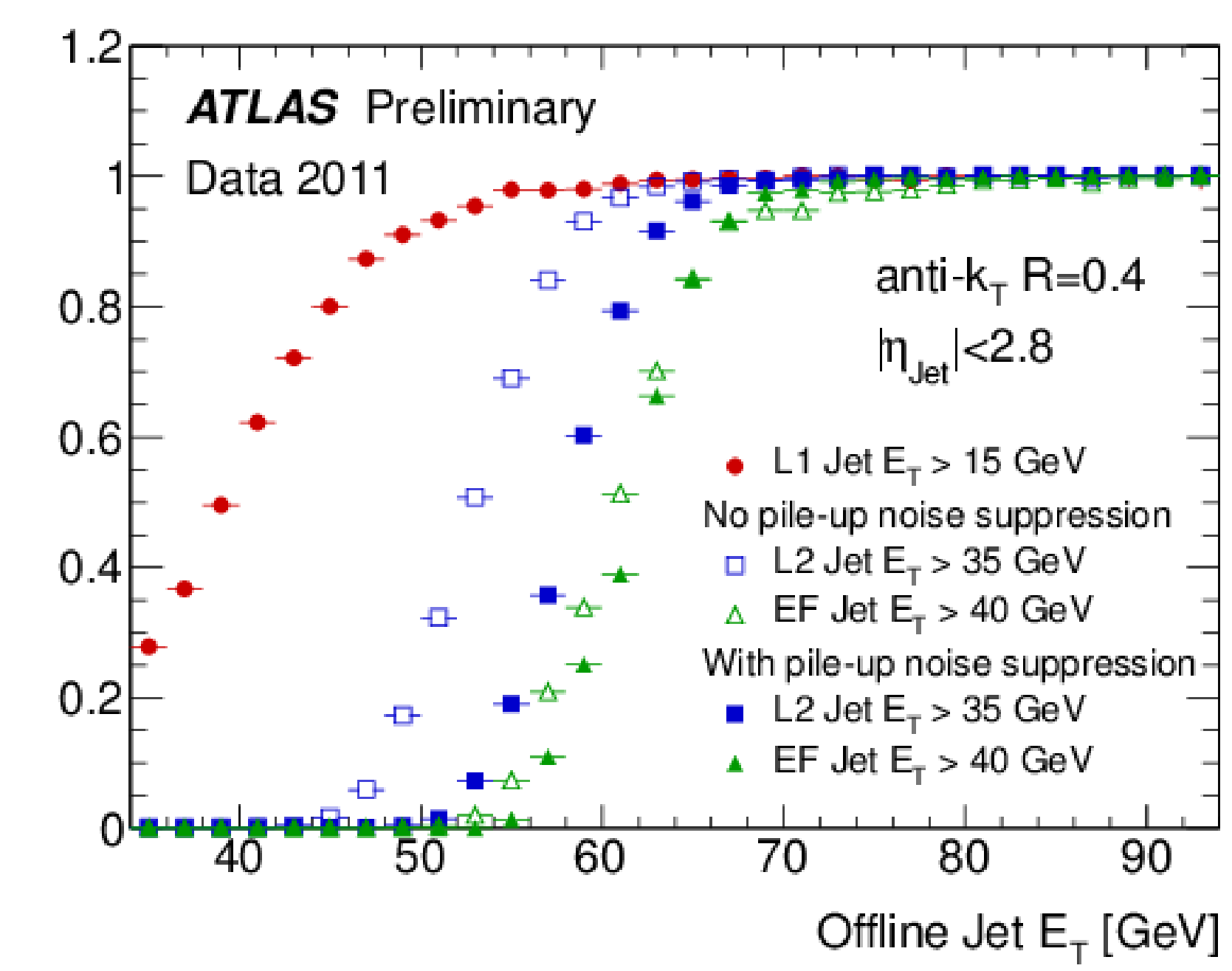

Trigger efficiency of an inclusive jet trigger chain $(\mathrm{L} 1 \rightarrow \mathrm{L} 2 \rightarrow \mathrm{EF})$ at the different trigger levels with respect to offline reconstructed ets, using the anti-k algorithm with $\mathrm{R}=0.4$. The thresholds at the different levels are tuned such that the trigger plateau is always reached at approximately the same offline jet $\mathrm{E}_{\mathrm{T}}$. The pile-up suppression has a larger effect at L2 compared to the EF, since the topological clusters used at EF already include some noise suppression.

\section{Jet Trigger in Heavy lon Collisions}

In $\mathrm{Pb}-\mathrm{Pb}$ collisions, centrality describes the central or tangential nature of the heavy ion collisions More central collisions involve a larger number of colliding nucleons and thus a larger number of created particles. In central collisions, the creation of a hot, dense medium, the quark-gluon plasma, is predicted by QCD. Since higher particle multiplicity leads to a higher activity in the calorimeters, as a result, a decrease in trigger efficiency is expected with increased centrality of the collision. Dedicated underlying event subtraction algorithms are applied at the EF to correct the $\mathrm{EF}$ jet energies for underlying energy densities and thus reduce the effect of high detector occupancy on the trigger efficiency.

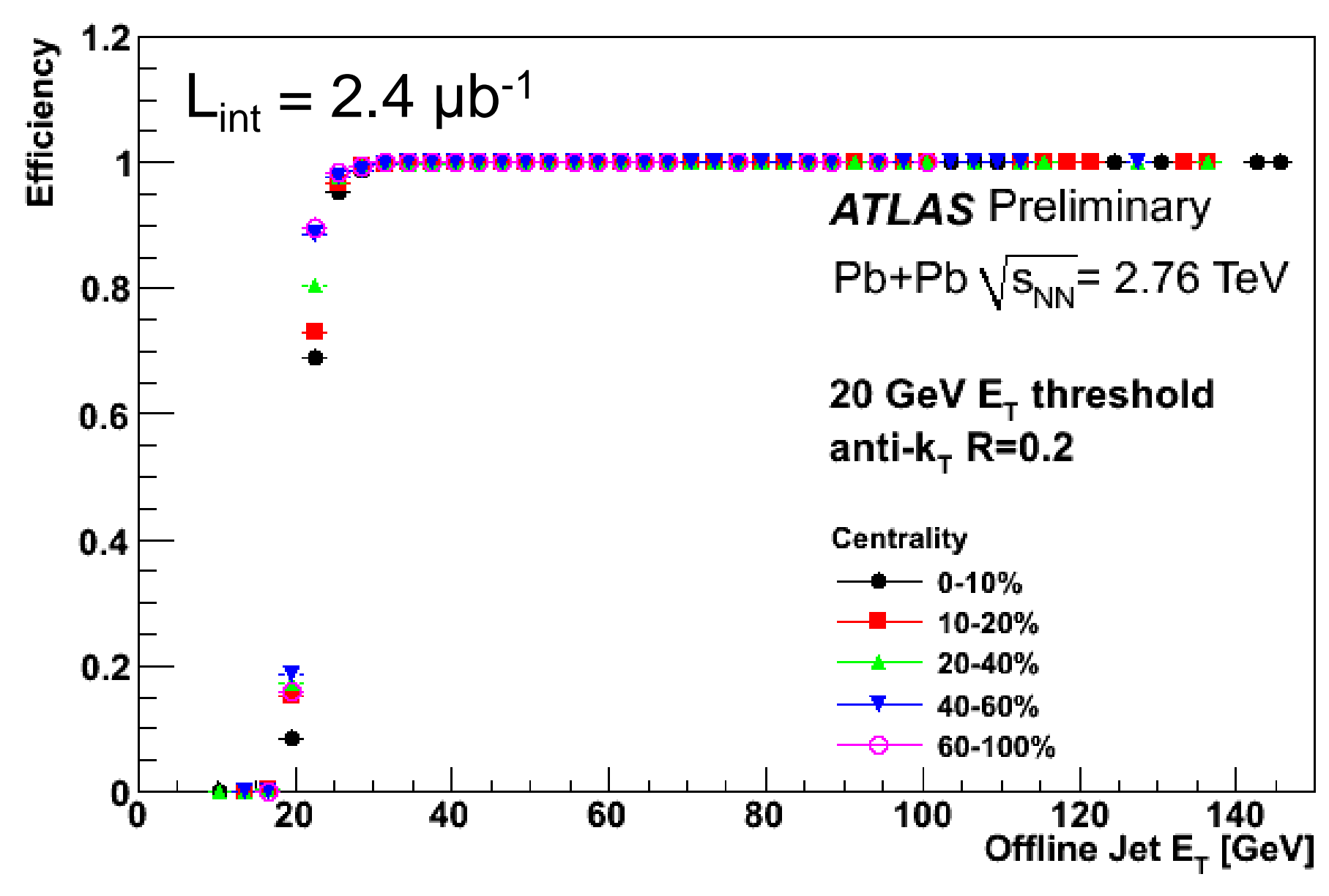

Efficiency of the primary HLT jet trigger for heavy ion collisions in 2011. The L1 seed of this trigger required $\mathrm{E}_{\mathrm{T}}$ total $>10 \mathrm{GeV}$ and, at the $\mathrm{HLT}$ as well as offline, jets were identified with the anti- $\mathrm{k}_{\mathrm{t}}$ algorithm with $R=0.2$. The independence of the trigger efficiency on the centrality of the collisions is achieved by the high performance of the underlying event subtraction which corrects the calorimeter cell energies for the energy densities measured outside the reconstructed jets in the event.

\section{Summary}

The extensions to the ATLAS jet trigger system in 2011 - full calorimeter unpacking at EF, L1.5 trigger, dedicated pile-up and noise suppression, underlying event subtraction - have shown excellen performance during data taking with $\mathrm{p}-\mathrm{p}$ and $\mathrm{Pb}-\mathrm{Pb}$ collisions in 2011 and further improved the high performance of the jet trigger system. The improved performance especially in multi-jet events and in a high pile-up environment presents an important requirement for data-taking in 2012 